\title{
Fast Computation of the Inverse CMH Model
}

\author{
Umesh D. Patel* and Edward Della Torre** \\ *Goddard Space Flight Center, Code 564, Greenbelt, MD 20771 USA, e-mail:upatel@pop500.gsfc.nasa.gov \\ **George Washington University, DC 20052 USA, e-mail:dellator@seas.gwu.edu
}

\begin{abstract}
A fast computational method based on differential equation approach for inverse DOK model has been extended for the inverse CMH model. Also, a cobweb technique for calculating the inverse CMH model is also presented. The two techniques are differed from the point of view of fexibility and computation time.
\end{abstract}

\section{INTRODUCTION}

The CMH model is a moving Preisach model in which the reversible component of the magnetization is state-dependent. Unlike the DOK model, it accurately predicts the variation in zero-field susceptibility with magnetization. It has not been used much in the past because the DOK model, which is much easier to calculate, is accurate enough in most cases to describe the magnetization process and a fast simple algorithm was not available.

Two fast techniques based on the differential equation method and the cobweb method for calculating the $\mathrm{CMH}$ model have been presented in [1]. The speed increase of the two techniques results from computing the magnetization by simply adding the change in magnetization due to any change in applied field to the previously computed magnetization, instead of integrating over the Preisach plane at each step of the computation.

Ferromagnetic actuators are used for active control in many automotive, industrial, and aerospace applications. Peizoceramic, Shape memory alloy, and Terfenol-D are more widely used ferromagnetic materials, even though they display the hysteretic behaviour at large drive levels. For high accuracy or micropositioning applications, an accurate compensator based controller can improve the performance of ferromagnetic actuators [2-4]. The fast inverse $\mathrm{CMH}$ model based computational methods presented here could be used to compensate the hysteretic effects.

\section{THE INVERSE CMH MODELS}

The inverse $\mathrm{CMH}$ model in functional form is given by

$$
\begin{aligned}
& \frac{d h}{d M}=\chi^{-1}\left(M, h, m_{I}, a_{+}, a_{-}, \frac{d a_{+}}{d h}, \frac{d a_{-}}{d h}\right) \\
& \frac{d a_{+}}{d h}=\phi_{a^{+}}\left(M, h, m_{l}\right) \\
& \frac{d a_{-}}{d h}=\phi_{d-}\left(M, h, m_{l}\right) \\
& \frac{d m_{t-}}{d M}=\phi_{l}\left(M, h, m_{l}\right)
\end{aligned}
$$

The function $\Phi_{I}$ can be defined as

$$
\Phi_{1}=\frac{d m_{1}}{d M}=\frac{d m_{l}}{d h} \frac{d h}{d M}=\chi_{l} \chi^{-1}
$$

The signal flow for an inverse CMH model is shown in Fig. 1.

The $\mathrm{CMH}$ model is implemented based on the cobweb grid systems for irreversible magnetization and $a_{+}$and $a_{\text {. }}$ variables for the reversible magnetization [1]. A detailed algorithm for inverse $\mathrm{CMH}$ model using cobweb approach is developed. Details of the algorithm will be presented in the final paper.

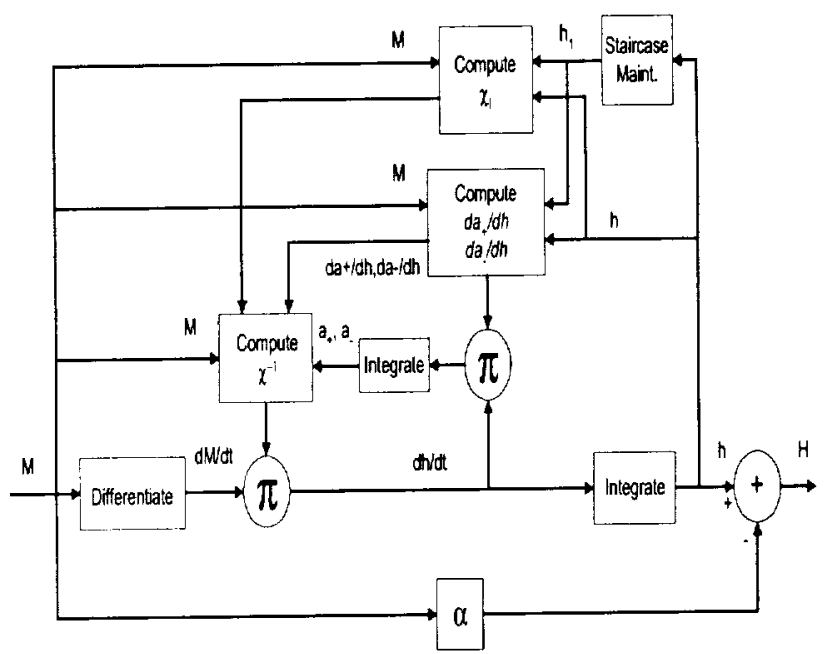

Fig. 1. The inverse CMH model signal flow.

Simulation results for both methods to compute the inverse $\mathrm{CMH}$ model are presented and compared in terms of computation time and flexibility.

\section{REFERENCES}

[1]. U. Patel and E. Della Torre, "Fast Computation of CMH Model," Submitted to COMPUMAG, France, July 2001.

[2]. A. Reimers and E. Della Torre, "Fast Preisach based magnetization model and fast inverse hysteresis model," IEEE Trans. Magn., 34, Nov. 1998, pp.3857-3866.

[3] E. Della Torre, Magnetic Hysteresis, IEEE Press, Piscataway, NJ, 1999.

[4]. J. Shaefer and H. Janocha, "Compensation of hysteresis in solid-state actuators," Sens. Actuators, A:Physical, vol.49, no. 1-2, pp.97-102, June 1995. 